\title{
A Local Mobility Model for Ultra-Thin DGSOI nMOSFETs
}

\author{
Andreas Schenk \\ Integrated Systems Lab., ETH Zürich, Gloriastr. 35, CH-8092 Zürich, Switzerland \\ schenk@iis.ee.ethz.ch
}

\begin{abstract}
The derivation of a local mobility model for symmetrical ultra-thin DGSOI nMOSFETs is outlined. A local-field variant is found to reproduce the dependencies of the quantummechanical mobility on silicon slab thickness and normal field with a maximum error of $10 \%$. The model can be used with the density-gradient approach.
\end{abstract}

\section{Introduction}

Currently there is strong interest in ultra-thin DGSOI transistors due to their potential perspectives for better scalability, higher performance, and reduced short-channel effects. A theoretical enhancement of the low-field mobility for silicon slab thicknesses $t_{\mathrm{Si}} \approx 10 \mathrm{~nm}$ was shown to be unique to symmetrical variants [1]. TCAD application with DESSIS_ISE of an integrated quantum-mechanical (q.m.) mobility model based on a 1D Schrödinger/Poisson solver was demonstrated in $[1,2]$. However, limited numerical robustness and considerable CPU times call for a simple local model. Whereas local mobility models for single-gate MOSFETs [3, 4] are widely used in the TCAD community, a similar model for ultra-thin DGSOI devices is still missing. The aim of this paper is to outline the derivation of such a model, to point out the particular difficulties involved, and to discuss its accuracy compared to the q.m. model.

\section{Model Restrictions and Development Strategy}

The complex physics and the dependence on various parameters require a step-by-step development of such a model. In a first step which is outlined in this paper we neglect all kinds of Coulomb scattering [5] and restrict ourselves to symmetrical DGSOI nMOSFETs. We assume equal roughness of both interfaces with parameters that reproduce the measured universal $\mu_{\text {eff }}\left(E_{\text {eff }}\right)$ dependence of bulk MOSFETs [6] ( $\Delta_{1,2}=0.32 \mathrm{~nm}$, $L_{1,2}=1.5 \mathrm{~nm}$ ). Electron-phonon coupling constants and effective phonon energies are assumed to keep their bulk values even for the thinnest silicon slabs. We neglect poly-silicon effects (i.e. actually assume metal-gate boundary conditions) and also disregard longitudinal quantum effects $\left(L_{\mathrm{G}} \geq 20 \mathrm{~nm}\right)$. Nonparabolicity $(\alpha=0.5 / \mathrm{eV})$ is included, because it has a $10 \%$ effect on the mobility. It turns out that the effective mobility only weakly depends on the oxide thickness $t_{\mathrm{ox}}$. Therefore, we omit this dependence in a first step and use $t_{\mathrm{ox}}=0.6 \mathrm{~nm}$ throughout the simulations. The subsequent inclusion of this dependence in one parameter of the local model would be straightforward. From the simulated $\mu_{\text {eff }}\left(E_{\text {eff }}\right)$ curves in the $t_{\mathrm{Si}}$-range from $1 \mathrm{~nm}$ to $15 \mathrm{~nm}$ (Fig. 1) we extract the low-field parameter $\mu_{\text {low }}\left(t_{\mathrm{Si}}\right)$ as function of silicon slab thickness. This dependence is shown on the right-hand side of Fig. 1. One observes 

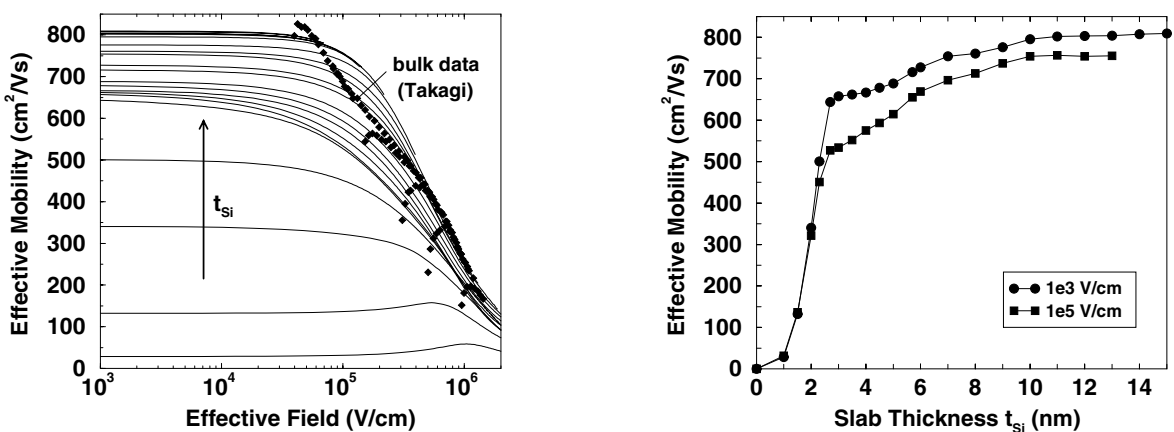

Figure 1: Left: Effective mobility as function of effective field $E_{\text {eff }}$ for Si slab thicknesses $t_{\mathrm{Si}}$ in the range from $1 \mathrm{~nm}$ to $15 \mathrm{~nm}$ (no Coulomb scattering). The values of $t_{\mathrm{Si}}$ are listed in Table 1. Right: Effective mobility versus slab thickness $t_{\mathrm{Si}}$ for two values of the effective field. A cubic-spline fit to the upper curve yields the parameter $\mu_{\text {low }}\left(t_{\mathrm{Si}}\right)$ for the local model.

\begin{tabular}{|l|l|l|l|l|l|l|l|}
\hline$t_{\mathrm{Si}}(\mathrm{nm})$ & 1.0 & 1.5 & 2.0 & 2.3 & 2.7 & 3.0 & 3.5 \\
\hline$\mu_{\text {low }}\left(\mathrm{cm}^{2} / \mathrm{Vs}\right)$ & 28.81 & 132.5 & 340.65 & 500.44 & 643.51 & 657.29 & 661.92 \\
\hline \hline$t_{\mathrm{Si}}(\mathrm{nm})$ & 4.0 & 4.5 & 5.0 & 5.7 & 6.0 & 7.0 & 8.0 \\
\hline$\mu_{\text {low }}\left(\mathrm{cm}^{2} / \mathrm{Vs}\right)$ & 666.5 & 678.3 & 688.29 & 715.6 & 727.37 & 753.75 & 760.81 \\
\hline \hline$t_{\mathrm{Si}}(\mathrm{nm})$ & 9.0 & 10 & 11 & 12 & 13 & 14 & 15 \\
\hline$\mu_{\text {low }}\left(\mathrm{cm}^{2} / \mathrm{Vs}\right)$ & 775.98 & 795.3 & 801.81 & 803.30 & 803.6 & 807.13 & 809.21 \\
\hline
\end{tabular}

Table 1: Low-field values of $\mu_{\mathrm{eff}}\left(t_{\mathrm{Si}}\right)$ from which the cubic-spline function $\mu_{\text {low }}\left(t_{\mathrm{Si}}\right)$ can be generated.

the region of geometrical confinement $\left(t_{\mathrm{Si}}<3 \mathrm{~nm}\right)$ with a rapid decay of the mobility and a broad maximum around $t_{\mathrm{Si}} \approx 10 \mathrm{~nm}$, where the mobility is enhanced by $10 \%$ $-15 \%$ in virtue of interference between the two channels. The remaining structure around $t_{\mathrm{Si}} \approx 7 \mathrm{~nm}$ is real (mesh-refinement effects are much smaller). For the parameterization of this involved $\mu_{\text {low }}\left(t_{\mathrm{Si}}\right)$-dependence any analytical ansatz fails; therefore a cubic-spline interpolation based on the discrete data points is the best alternative. This spline can be generated from Table 1 . The $\mu_{\mathrm{eff}}\left(E_{\mathrm{eff}}\right)$-dependence with its $t_{\mathrm{Si}^{-}}$ independent high-field limit for $t_{\mathrm{Si}}>5 \mathrm{~nm}$ (Fig. 1) suggests a simple local model of the form $\mu\left(E_{\perp}, t_{\mathrm{Si}}\right)=\mu_{\text {low }}\left(t_{\mathrm{Si}}\right) /\left(1+\left(E_{\perp} / E_{\text {ref }}\right)^{b_{E}}\right)$ with two $t_{\mathrm{Si}}$-dependent parameters $E_{\text {ref }}$ and $b_{E}$. In order to find a suitable parameterization of these functions, the only promising way is to use $\mu_{\text {eff }}\left(E_{\text {eff }}\right)$ instead of some $\mu_{\text {loc }}\left(E_{\perp, \text { loc }}\right)$ from an inner point, e.g. at the surface. Therefore, the functions $E_{\text {ref }}\left(t_{\mathrm{Si}}\right)$ and $b_{E}\left(t_{\mathrm{Si}}\right)$ are determined iteratively by non-linear curve fitting to each curve on the left-hand side of Fig. 1. As a result, $E_{\text {ref }}\left(t_{\mathrm{Si}}\right)$ and $b_{E}\left(t_{\mathrm{Si}}\right)$ become available in form of algebraic expressions.

\section{Local-Field versus Local-Density Model}

If the parameterization obtained in this way is applied to the local-field model, a larger error must be expected for the on-current. Since the $E_{\perp}$-field in the center is always zero, the local mobility will be pinned to its low-field value there, whereas the q.m. mobility drops with increasing gate voltage (right-hand side of Fig. 2). The charge density, on the other hand, has a substantial value in the center of ultra-thin DGSOIs. The resulting error for the on-current can be seen from the left-hand side of Fig. 2. To 

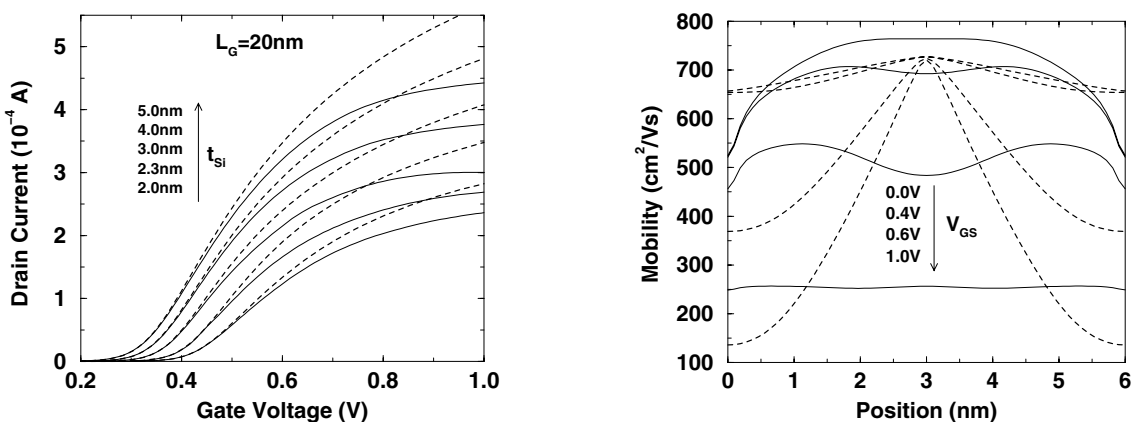

Figure 2: Left: Transfer characteristics at $V_{\mathrm{DS}}=10 \mathrm{mV}$ for $2 \mathrm{~nm} \leq t_{\mathrm{Si}} \leq 6 \mathrm{~nm}$. Right: Evolution (from top to bottom) of the mobility profiles across the slab with increasing $V_{\mathrm{GS}}$ in the case of $t_{\mathrm{Si}}=6 \mathrm{~nm}$. Solid lines: q.m. model, dashed lines: local-field model.

overcome this problem, a parameterization of the mobility in terms of the density was taken into consideration. The resulting local-density model $\mu\left(n, t_{\mathrm{Si}}\right)=\mu_{\text {low }}\left(t_{\mathrm{Si}}\right) /(1+$ $\left.\left(n / n_{\text {ref }}\right)^{b_{n}}\right)$ with the local 3D density $n$ was developed on the same lines as the localfield model. Starting point were the simulated functions $\mu_{\mathrm{eff}}\left(n_{\mathrm{av}}\right)$ for the same $t_{\mathrm{Si}^{-}}$ range as in Fig. 1. Iterative non-linear curve fitting yielded the two parameters $n_{\text {ref }}\left(t_{\mathrm{Si}}\right)$ and $b_{n}\left(t_{\mathrm{Si}}\right)$. The mobility profiles of the local-density model have minima exactly where the density has its maxima (immediately obvious from the formula of the localdensity model). This results in an underestimation of the on-current with an amount

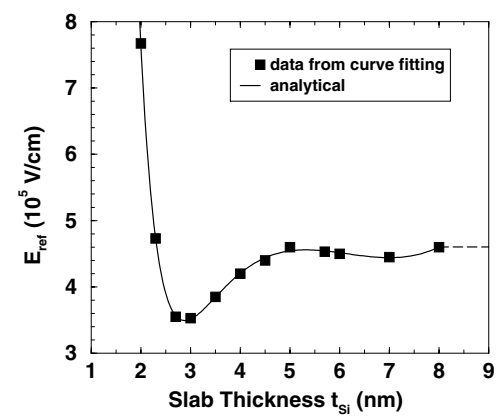

\begin{tabular}{|l|l||l|l|}
\hline$c_{0}$ & $-6.24142 \mathrm{e} 6$ & $c_{-4}$ & $-8.01531 \mathrm{e} 7$ \\
\hline$c_{-1}$ & $3.5754 \mathrm{e} 7$ & $c_{1}$ & $9.49686 \mathrm{e} 5$ \\
\hline$c_{-2}$ & $-1.14565 \mathrm{e} 8$ & $c_{2}$ & $-9.62632 \mathrm{e} 4$ \\
\hline$c_{-3}$ & $1.69859 \mathrm{e} 8$ & $c_{3}$ & $4.44057 \mathrm{e} 3$ \\
\hline
\end{tabular}

Figure 3: Final fit of the function $E_{\mathrm{ref}}\left(t_{\mathrm{Si}}\right)$ (solid line) with coefficients $c_{n}$ given in the table.

which is even stronger than the overestimation by the local-field model. For the purpose of a TCAD model, transfer characteristics at $V_{\mathrm{DS}}=10 \mathrm{mV}$ were then used in order to re-adjust the parameters $E_{\mathrm{ref}}\left(t_{\mathrm{Si}}\right)$ and $b_{E}\left(t_{\mathrm{Si}}\right)$ for a close fit to the currents. The $t_{\mathrm{Si}^{-}}$ dependence of these parameters greatly simplifies $\left(b_{E}\left(t_{\mathrm{Si}}\right)\right.$ can be taken as a constant 1.7) and the maximum relative error becomes $10 \%$ for $t_{\mathrm{Si}}>2 \mathrm{~nm}$ (Fig. 4). The final model reads

$\mu\left(E_{\perp}, t_{\mathrm{Si}}\right)=\frac{\mu_{\text {low }}\left(t_{\mathrm{Si}}\right)}{1+\left[E_{\perp} / E_{\mathrm{ref}}\left(t_{\mathrm{Si}}\right)\right]^{1.7}}$ with

$E_{\mathrm{ref}}(d)=C(d) \Theta(8-d)+C(8) \Theta(d-8), \quad C(d)=\sum_{n=-4}^{+3} c_{n} d^{n}$

where $d=t_{\mathrm{Si}} / n m$. The coefficients $c_{n}$ are given in the table of Fig. 3 . 

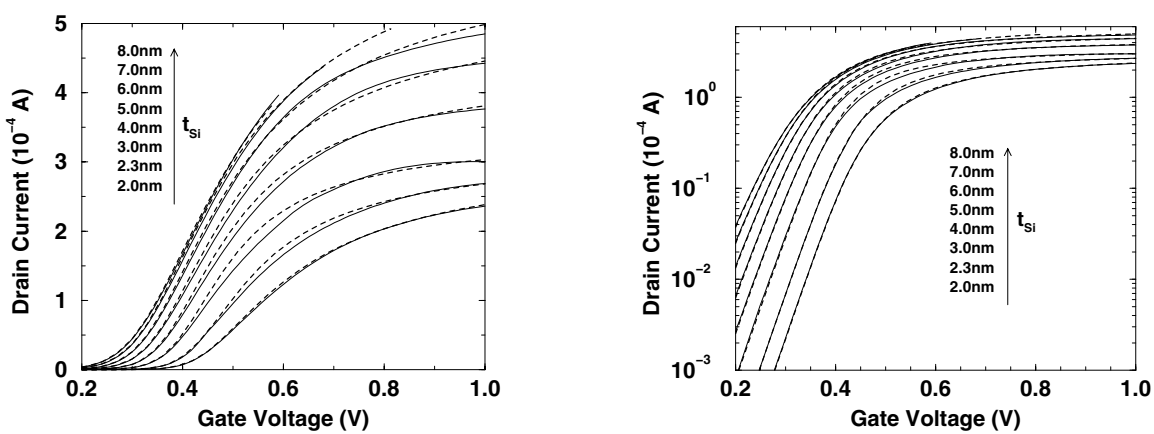

Figure 4: Transfer characteristics at $V_{\mathrm{DS}}=10 \mathrm{mV}$ for $2 \mathrm{~nm} \leq t_{\mathrm{Si}} \leq 8 \mathrm{~nm}$ with the q.m. model (solid lines) and the final local-field model (dashed lines). Left: lin-lin scale, right: lin-log scale.

\section{Conclusion}

A local-field model for the mobility of symmetrical ultra-thin DGSOI nMOSFETs is demonstrated to reproduce transfer characteristics with a maximum error of $10 \%$ for silicon slab thicknesses $t_{\mathrm{Si}}>2 \mathrm{~nm}$. The accuracy is limited by the general impossibility to map a nonlocal q.m. quantity to the corresponding local classical quantity. As in the case of established local mobility models for single-gate transistors, the usability of the presented model comes from the crossing of q.m. and local mobility profiles at points of high electron and current densities. In single-gate transistors these points coincide with the single centroids of the relatively sharp density distributions. In ultra-thin DGSOI devices volume inversion leads to a high density in the middle of the silicon slab, too, which makes the method less straightforward. Besides negligible CPU time the actual advantage of the local model is that a Schrödinger-Poisson solver becomes dispensable, because only the q.m. normal field is needed. The latter can already be obtained by the density-gradient approach.

\section{Acknowledgment}

The author is grateful for many valuable discussions with Dr. A. Wettstein (ISE AG Zurich) and for the financial support by Fujitsu Laboratories Ltd.

\section{References}

[1] A. Schenk and A. Wettstein, "Simulation of DGSOI MOSFETs with a Schrödinger-Poisson Based Mobility Model“, Proc. SISPAD 2002, pp. 21-24.

[2] A. Wettstein, "Quantum Effects in MOS Devices", PhD Thesis No. 13649, ETH Zrich, ISBN: 3-89649-566-6, Hartung-Gorre, 2000.

[3] S. A. Schwarz and S. E. Russek, "Semi-Empirical Equations for Electron Velocity in Silicon”, IEEE Trans. El. Dev., ED-30 (12), pp. 1629-1633, 1983.

[4] M. N. Darwish, J. L. Lentz, M. R. Pinto, P. M. Zeitzoff, T. J. Krutsick, and H. H. Vuong, "An Improved Electron and Hole Mobility Model for General Purpose Device Simulation", IEEE Trans. El. Dev., ED-44 (9), pp. 1529-1537, 1997.

[5] F. Gamiz and M. V. Fischetti, "Remote Coulomb Scattering in MOSFETs: Screening by Electrons in the Gate", APL 83 (23), pp. 4848-4850, (2003).

[6] S. Takagi, A. Toriumi, M. Iwase, and H. Tango, "On the Universality of Inversion Layer Mobility in Si MOSFET's”, IEEE Trans. El. Dev., ED-41 (12), pp. 2357-2368, 1994. 